\title{
Nonconventional MRI biomarkers for in vivo monitoring of pathogenesis in multiple sclerosis
}

\section{OPEN}

Ana C. Londoño, MD

Carlos A. Mora, MD

Correspondence to Dr. Mora:

cxmz@gunet.georgetown.edu

\section{ABSTRACT}

To date, biomarkers based on nonconventional MRI have not been standardized for diagnosis and follow-up of patients with multiple sclerosis (MS). The sequential monitoring of pathogenesis in MS by imaging of the normal appearing brain tissue is an important research tool in understanding the early stages of MS. In this review, we focus on the importance of deciphering the physiopathogenesis of the disease cascade in vivo based on imaging biomarkers that allow a correlation with immunohistochemistry and molecular biology findings in order to provide earlier clinical diagnosis and better individualization of treatment and follow-up in patients with MS. Among the nonconventional imaging techniques available, we remark on the importance of proton magnetic resonance spectroscopy imaging because of its ability to assist in the simultaneous evaluation of different events in the pathogenesis of MS that cannot be determined by conventional MRI. Nonconventional MRI and the use of novel contrast agents are expected to elucidate the process of neuroinflammation and excitotoxicity in vivo that characterizes MS, thus leading to more specific neuroprotective and immunomodulatory therapies and reducing progression toward disability. Neurol Neuroimmunol Neuroinflamm 2014;1:e45; doi: 10.1212/NXI.0000000000000045

\section{GLOSSARY}

${ }^{1} \mathrm{H}$-MRSI = proton magnetic resonance spectroscopy imaging; $\mathbf{A D}=$ Alzheimer disease; $\mathbf{B B B}=$ blood-brain barrier; $\mathbf{C I S}=$ clinically isolated syndrome; $\mathbf{c M R I}=$ conventional MRI; $\mathbf{C R}=$ creatine; $\mathbf{D T I}=$ diffusion tensor imaging; EDSS = Expanded Disability Status Scale; GLX = glutamate-glutamine complex; $\mathbf{m l}=$ myo-inositol; $\mathbf{M S}=$ multiple sclerosis; NAA $=\mathrm{N}$-acetylaspartate; $\mathbf{N A B T}=$ normal appearing brain tissue; $\mathbf{N A W M}=$ normal appearing white matter; $\mathbf{N M O}=$ neuromyelitis optica; PPMS = primary progressive multiple sclerosis; $\mathbf{R I S}=$ radiologically isolated syndrome; $\mathbf{R R M S ~ = ~}$ relapsing-remitting multiple sclerosis; SPMS = secondary progressive multiple sclerosis; $\mathbf{S W I}$ = susceptibility-weighted imaging; TNF- $\alpha=$ tumor necrosis factor $\alpha$.

As the most common nontraumatic cause of neurologic disability in early to middle adulthood, multiple sclerosis (MS) is a complex immune-mediated disorder of the CNS characterized by inflammation, demyelination, gliosis, axonal degeneration, and neuronal loss. It is estimated that worldwide close to 2.5 million individuals are affected by MS, and there are at least 400,000 patients diagnosed with MS in the United States. ${ }^{1}$ The estimated total direct and indirect cost of MS in the United States is close to $\$ 28$ billion each year, and the annual cost of care for individuals with MS, excluding cost of drugs, is on average 4.5 times that of healthy controls. This cost of care escalates in direct proportion to the increment in the Expanded Disability Status Scale (EDSS) score of affected individuals. Hence, stopping or reversing disease progression is a priority. This therapeutic endeavor is becoming a reality thanks to novel techniques in image analysis, in particular related to MRI, and to the advances in immunotherapy over the last 2 decades. In a redefinition of therapy goals with new modifying agents for MS, freedom from disease (characterized by absence of relapses, absence of disability, and absence of radiologic evidence of disease activity) is increasingly seen as the measure of treatment success. ${ }^{2}$ Here, we focus on the importance of achieving freedom from disease based on the appropriate use of currently available MRI techniques. A determining factor for a better

From the Neurological Institute of Colombia-INDEC and Link Diagnostico Digital (A.C.L.), Medellín, Colombia; and Department of Neurology (C.A.M.), Georgetown Multiple Sclerosis Center, MedStar Georgetown University Hospital, Washington, DC.

Go to Neurology.org/nn for full disclosures. Funding information and disclosures deemed relevant by the authors, if any, are provided at the end of the article. The Article Processing Charge was paid by the Department of Neurology of MedStar Georgetown University Hospital.

This is an open access article distributed under the terms of the Creative Commons Attribution-Noncommercial No Derivative 3.0 License, which permits downloading and sharing the work provided it is properly cited. The work cannot be changed in any way or used commercially. 
prognosis of MS is the elimination of progression of inflammation and neurodegeneration in an early stage of the disease. Hence, the decision to start and maintain treatment in patients with early forms of MS such as typical and atypical clinically isolated syndrome (CIS), radiologically isolated syndrome (RIS), and early relapsing-remitting MS (RRMS) has been a challenge for the neurologist in clinical practice. In fact, according to a survey regarding current MS practice patterns for CIS, RRMS, and RIS, US-based MS specialists considered RIS possibly indicative of clinically definite MS risk and remarked on the importance of MRI and clinical follow-up but were unlikely to recommend initiating treatment for RIS. ${ }^{3}$

The McDonald criteria of 2010 made it possible to diagnose MS with the first episode of disease, therefore reducing the number of events before definitive clinical diagnosis, which made it difficult to demonstrate any statistical differences between the treatments. ${ }^{4,5}$ In addition, clinical judgment in MS usually stems from the support provided by both clinical assessment and conventional MRI (cMRI) data. However, the dissociation between clinical presentation and cMRI findings is well-known, indicating that an increment in lesion load on cMRI may not necessarily be associated with clinical manifestations and that clinical manifestations may not present concomitantly with changes in cMRI. Therefore, it is imperative to consider additional methodology in clinical practice, such as the use of nonconventional MRI techniques for the analysis of biomarkers of MS activity. This methodology allows the determination of cerebral microstructural, functional, and biochemical abnormalities, as well as disruption of the blood-brain barrier (BBB), before these abnormalities could be detected by cMRI. This step could assist the clinician in tailoring early individual therapeutic interventions that may eliminate disease progression.

In order to choose an optimal and individualized therapy for patients with MS, it is important to understand the sequence of events that takes place in the pathogenesis of the disease, even though the precise role and time of intervention of several contributing pathogenic factors have not been clearly established. Among these factors is the disruption of the BBB allowing the influx and accumulation of lymphocytes into the meninges and perivascular spaces and most likely taking place in an early stage of the disease. These inflammatory infiltrates are composed of $\mathrm{T}$ lymphocytes, B lymphocytes, plasma cells (including macrophages), activated microglia, and dysfunctional astrocytes. ${ }^{6,7}$ The vulnerability of oligodendrocytes and neurons to injury may also be promoted by dysfunctional astrocytes ${ }^{8}$ and by oxidative stress induced by the production of free radicals and release of cellular iron deposits, leading to mitochondrial injury. ${ }^{6}$ These factors also include the role played by CNS macrophages mainly derived from blood monocytes and resident microglia. ${ }^{9}$ The role of the microglia entails a state of permanent surveillance and protection against any signal of aggression toward the homeostasis of the CNS. ${ }^{10}$

What ultimately triggers demyelination in MS still deserves further investigation. There is evidence supporting the existence of a "demyelinating factor," which could entail the combination of B-cell cytokines ${ }^{11}$ as supported by therapeutic effects of B-cell depletion. ${ }^{12}$ It has also been postulated that humoral- and complement-mediated responses may lead to demyelination and damage to the oligodendroglia and that axonal degeneration may occur in a nonuniform distribution during the acute phase of demyelination, even within demyelinating plaques in the brain of the same individual. ${ }^{6}$

Astrocytopathy should also be considered a crucial factor in the physiopathogenesis of MS given that astrocytes are involved in the development, regulation, and maintenance of the $\mathrm{BBB}^{13}$; influx of toxins and ions such as $\mathrm{K}^{+}$ and $\mathrm{Ca}^{2+}$; maintenance of the physiologic $\mathrm{pH}$; modulation of axonal action potential propagation; clearance of neurotransmitters, especially glutamate, in the extracellular space; and regulation of iron metabolism. ${ }^{14}$

Complete understanding of the cascade of events involved in the etiology and pathogenesis of MS is still a challenge that, once overcome, may allow earlier diagnosis and more efficient treatments. Current research may be on the verge of deciphering this cascade of events in vivo, with the support of in vitro studies, thanks to the development of animal models that recreate different phenotypes of disease (monophasic, relapsing-remitting, chronic-progressive, 
or chronic-relapsing disease depending on the animal strains and immunogens used) and to the availability of advanced MRI sequences that allow evaluation before and during progression of disease in individualized animals. ${ }^{15}$ In spite of the outcome of previous studies based on animal models and human autopsy data, it is crucial to document the sequence of pathologic events leading to MS in humans in vivo in order to validate new biomarkers of disease activity. Therefore, application of nonconventional MRI techniques to determine an active pathologic process in macroscopically normal appearing brain tissue (NABT) may lead to a better understanding of the $\mathrm{BBB}$ impairment in MS. ${ }^{16}$ This may also include better visualization of the white matter microstructure using diffusion tensor imaging (DTI), mapping of brain iron deposits by susceptibility-weighted imaging (SWI), and evaluation of the cerebral biochemical state during cascade of the inflammatory process, including demyelination and mitochondrial and astrocytic dysfunction, by proton magnetic resonance spectroscopy imaging $\left({ }^{1} \mathrm{H}-\mathrm{MRSI}\right)$ (table).

Disruption of the BBB in NABT has been suspected to begin before the onset of demyelination and infiltration of leukocytes in MS lesions. ${ }^{16}$ Evidence has shown extensive increase in BBB permeability in periventricular normal appearing white matter (NAWM), especially in areas quick to develop lesions, as well as in the thalamic nuclei in patients affected by RRMS.${ }^{16}$ However, there has been no evidence that correlates alteration in $\mathrm{BBB}$ with age, EDSS, and disease duration. In progressive MS, the inflammatory process remains entrapped within the CNS despite a closed or repaired $\mathrm{BBB}$ and in the presence of accumulated lymph follicles within the meninges. ${ }^{17,18,6}$ In these subpial lesions, diffusible factors from the CSF may play a role in the significant presence of activated microglia. ${ }^{10}$ Also, it has been shown that immunomodulatory therapy has the ability to repair the dysfunction of the BBB in MS. ${ }^{16}$ To our knowledge, evidence of $\mathrm{BBB}$ dysfunction in NABT other than the presence of contrastenhancing lesions has not been reported in CIS or RIS. Whether BBB dysfunction or infiltration of circulating autoreactive $\mathrm{T}$ cells is a requirement for the development of MS lesions remains controversial. ${ }^{16}$ Disruption of the $\mathrm{BBB}$ may be induced by the release of matrix metalloproteinase-9 from pericytes and CSF T cells. ${ }^{19,20}$ In pathologic conditions,

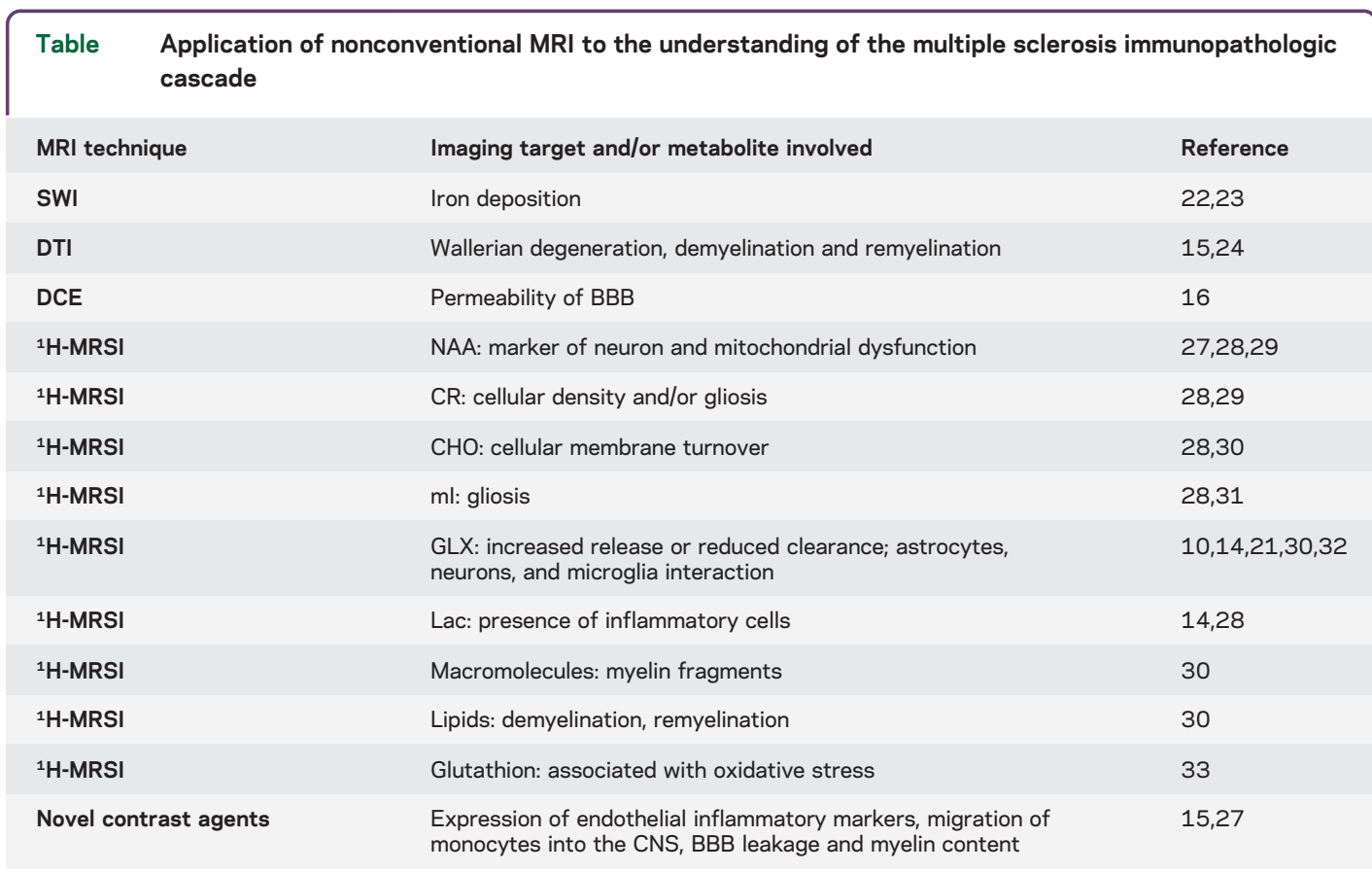

Abbreviations: ${ }^{1} \mathrm{H}-\mathrm{MRSI}=$ proton magnetic resonance spectroscopy imaging; $\mathrm{BBB}=$ blood-brain barrier; $\mathrm{CHO}=$ choline; $\mathrm{CR}$ = creatine; DCE = dynamic contrast enhancement; DTI = diffusion tensor imaging; GLX = glutamate-glutamine complex Lac = lactate; $\mathrm{ml}=$ myo-inositol; $\mathrm{NAA}=\mathrm{N}$-acetylaspartate; $\mathrm{SWI}=$ susceptibility-weighted imaging. 
astrocytes and mainly microglia release large amounts of tumor necrosis factor $\alpha$ (TNF $\alpha)$, which can potentiate glutamate-mediated cytotoxicity on astrocytes. ${ }^{21}$

The SWI sequence and quantitative susceptibility mapping allow the visualization of iron deposits in the CNS in the forms of deoxyhemoglobin, ferritin, or hemosiderin. ${ }^{22,23}$ Accumulation of iron in oligodendrocytes increases with aging and disease duration, eventually contributing to further cell injury due to oxidative stress and incremental mitochondrial damage. In addition, the release of iron from oligodendrocytes is more prominent in progressive disease than in the relapsing form. ${ }^{7}$ The in vivo determination of iron accumulation, with follow-up starting right after diagnosis, could become a useful biomarker to identify disease progression in its early stage.

DTI evaluates the motion of water molecules in tissues. Histologic studies have suggested that changes seen in the NAWM by DTI could result from wallerian degeneration. ${ }^{24}$ Abnormalities in diffusion have been reported in all phenotypes of MS, including CIS, and in spinal tissue of patients with MS and neuromyelitis optica (NMO). ${ }^{24}$ DTI allows for differentiation between axonal changes and demyelination even in NAWM. ${ }^{24,25}$ Since MS could be mistaken for relapsing NMO, DTI differentiates between spinal cord abnormalities in MS and $\mathrm{NMO}$, leading to early diagnosis and treatment. ${ }^{24,25}$ Increased mean diffusivity in white matter that communicates with spinal cord or optic nerve tracts in patients with NMO is believed to result from secondary degeneration due to lesions primarily located in the spinal cord or the optic nerve, respectively. ${ }^{26}$

The ${ }^{1} \mathrm{H}$-MRSI sequence allows an in vivo evaluation of different cerebral metabolites associated with cellular and functional processes. Among the metabolites determined with this technique are the following: (1) $\mathrm{N}$-acetylaspartate (NAA), which is synthesized in the neuronal mitochondria and is considered a marker of neuronal body and axonal integrity depending on transient (mitochondrial dysfunction) or permanent reduction of NAA (irreversible neuronal and/or axonal injury)..$^{27,28}$ In fact, decreased brain $\mathrm{NAA} /$ creatine $(\mathrm{CR})$ ratio, indicating brain metabolic abnormalities suggestive of axonal damage, has been recently reported in patients with RIS, ${ }^{29}$ (2) CR, which represents cellular metabolism and energy systems and therefore reflects changes in cellular density and/or gliosis. ${ }^{28}$ (3) Choline, which is a marker of cellular membrane turnover of phospholipids synthesis and degradation and correlates with phospholipid release during active demyelination. 28,30 (4) Myo-inositol (mI), which is synthesized in glial cells and has been associated with gliosis. ${ }^{28}$ The $\mathrm{mI} / \mathrm{NAA}$ ratio in NAWM has been reported to be a strong cross-sectional predictor of brain-volume loss and clinical disability evolution in patients with CIS, RRMS, secondary progressive MS (SPMS), and primary progressive MS (PPMS). ${ }^{31}$ (5) The glutamate-glutamine complex (GLX). Glutamate is the major excitatory neurotransmitter in the CNS regulated by the astrocytes in interstitial space ${ }^{14}$ and may be produced and released by activated leukocytes, macrophages, and microglial cells. ${ }^{30}$ Glutamate release and clearance is determined directly by the interaction between neurons and astrocytes and indirectly by microglia-released TNF $\alpha .{ }^{10}$ In a population of patients with different forms of MS, Azevedo et al. ${ }^{32}$ found that high concentrations of glutamate correlated with reduction of NAA and cerebral volume and decline of performance on the Multiple Sclerosis Functional Composite and the Paced Auditory Serial Addition Test-3. (6) Lactate, the presence of which may reflect metabolic disorders, especially mitochondrial dysfunction and/or presence of inflammatory cells. $^{28,14}$ (7) Glutathione, which is useful for the evaluation of oxidative stress and has been found to be reduced in patients with SPMS. ${ }^{33}$ (8) Lipids, which have been found in NAWM before the development of demyelinating plaques, have been reported in association with remyelination, and have been identified in NAWM of patients with PPMS. ${ }^{30}$ (9) Macromolecules, which may correspond to markers of myelin fragments. ${ }^{30}$

The extended use of ${ }^{1} \mathrm{H}$-MRS in clinical settings has been hampered by its technical demands. ${ }^{30}$ However, because of its ability to assist in simultaneous evaluation of different events involved in MS pathogenesis that cannot be determined by cMRI, ${ }^{1} \mathrm{H}-\mathrm{MRSI}$ could become an important tool to decipher the sequence of the immunologic cascade and to 
evaluate the response to new disease-modifying agents, including neuroprotectants.

The combined use of the abovementioned nonconventional MRI biomarkers could lead to a better understanding of different aspects of the disease process and play a key role in early diagnosis and prognosis of the disease, assessment of therapeutic response, and the understanding of different MS phenotypes. ${ }^{34}$ For instance, in studies of the early-onset Alzheimer disease (AD) PSEN1 E280A mutation, ${ }^{1} \mathrm{H}-\mathrm{MRSI}$ made it possible to establish a framework that helped identify mutation carriers, controls, asymptomatic patients with metabolic changes suggestive of subclinical $\mathrm{AD}$, and symptomatic patients. ${ }^{35}$

In the evaluation of patients with MS, the heterogeneity of results reported in different studies using different MRI techniques could have been related to differences in disease stage, disease phenotype, effect of pharmacologic intervention, quantification method, and the cerebral region chosen for evaluation. ${ }^{30}$ Cortical lesions and volume loss should not be overlooked since, on top of preceding the finding of white matter lesions by cMRI, they may also be associated with clinical disability, cognitive impairment, and progression of disease. The introduction of new pulse sequences, high-resolution imaging, and increased field strengths has led to an early detection of cortical damage in patients with MS. ${ }^{36}$ Contrast agents that have offered insight into the pathogenesis of MS in vivo include (1) iron nanoparticles and microparticles that identify mechanisms of expression of endothelial inflammatory markers and VCAM-1, follow the migration of monocytes into the CNS, and help target specific immune cells and oligodendrocytes; and (2) gadoliniumbased agents useful in evaluation of myelin content, enzyme activity present in active lesions, and BBB leakage. ${ }^{15,27}$

In conclusion, current and advanced MRI technology may allow the determination of lesion biomarkers in NABT, thus increasing the ability to modify the definition of disease activity, establish new diagnostic criteria, provide more assertive prognosis, and compare response to specific treatments more accurately. From a clinical standpoint, this step may help to lower the incidence of significant disability among patients with MS in the future by facilitating early diagnosis and early individualized application of specific immunomodulatory and/or neuroprotective treatments.

\section{AUTHOR CONTRIBUTIONS}

Dr. Londoño: drafting/critically revising the manuscript, study concept or design. Dr. Mora: drafting/critically revising the manuscript.

\section{ACKNOWLEDGMENT}

The authors thank Dr. Gholam Motamedi and Dr. John Richert for review of the manuscript.

\section{STUDY FUNDING}

No targeted funding reported.

\section{DISCLOSURE}

A.C. Londoño reports no disclosures. C.A. Mora is a member of the Data \& Safety Monitoring Board for a NINDS/NIH study. Go to Neurology. org/nn for full disclosures.

Received June 25, 2014. Accepted in final form October 3, 2014.

\section{REFERENCES}

1. Miller AE, Rhoades RW. Treatment of relapsing-remitting multiple sclerosis: current approaches and unmet needs. Curr Opin Neurol 2012;25(suppl 1):S4-S10.

2. Fox EJ, Rhoades RW. New treatments and treatment goals for patients with relapsing-remitting multiple sclerosis. Curr Opin Neurol 2012;25(suppl 1):S11-S19.

3. Tornatore C, Phillips JT, Khan O, Miller AE, Barnes CJ. Practice patterns of US neurologists in patients with CIS, RRMS, or RIS. Neurol Clin Pract 2012;2:48-57.

4. Montalban X, Tintore M, Swanton F, et al. MRI criteria for MS in patients with clinically isolated syndromes. Neurology 2010;74:427-434.

5. Milo R, Miller A. Revised diagnostic criteria of multiple sclerosis. Autoimmun Rev 2014;13:518-524.

6. Lassmann H. Multiple sclerosis: lessons from molecular neuropathology. Exp Neurol Epub 2013 Dec 14. doi: 10.1016/j.expneurol.2013.12.003.

7. Lassmann H. Mechanisms of white matter damage in multiple sclerosis. Glia 2014;62:1816-1830. doi: 10.1002/glia.22597.

8. Brosnan CF, Raine CS. The astrocyte in multiple sclerosis revisited. Glia 2013;61:453-465.

9. Yamasaki R, Lu H, Butovsky O, et al. Differential roles of microglia and monocytes in the inflamed central nervous system. J Exp Med 2014;211:1533-1549. doi: 10.1084/ jem20132477.

10. Ransohoff RM, Perry VH. Microglial physiology: unique stimuli, specialized responses. Annu Rev Immunol 2009;27: 119-145. doi: 10.1146/annurev.immunol.021908.132528.

11. Lisak RP, Benjamins JA, Nedelkoska L, et al. Secretory products of multiple sclerosis B-cells are cytotoxic to oligodendroglia in vitro. J Neuroimmunol 2012;246:85-95.

12. Hauser SL, Waubant E, Arnold DL, et al. B-cell depletion with rituximab in relapsing-remitting multiple sclerosis. $\mathrm{N}$ Engl J Med 2008;358:676-688.

13. Alvarez JI, Katayama T, Prat A. Glial influence on the blood brain barrier. Glia 2013;61:1939-1958.

14. Lundgaard I, Osorio MJ, Kress BT, Sanggaard S, Nedergaard M. White matter astrocytes in health and 
disease. Neuroscience 2014;276:161-173. doi: 10.1016/j. neuroscience. 2013.10.050.

15. Nathoo N, Yong VW, Dunn JF. Understanding disease processes in multiple sclerosis through magnetic resonance imaging studies in animal models. Neuroimage Clin 2014; 4:743-756. doi: 10.1016/j.nicl.2014.04.011.

16. Cramer SP, Simonsen H, Frederiksen JL, Rostrup E, Larssor HB. Abnormal blood-brain barrier permeability in normal appearing white matter in multiple sclerosis investigated by MRI. Neuroimage Clin 2013;4:182-189.

17. Hochmeister $S$, Grundtner R, Bauer J, et al. Dysferlin is a new marker for leaky brain blood vessels in multiple sclerosis. J Neuropathol Exp Neurol 2006;65:855-865.

18. Serafini B, Rosicarelli B, Magliozzi R, Stigliano E, Aloisi F. Detection of ectopic B-cell follicles with germinal centers in the meninges of patients with secondary progressive multiple sclerosis. Brain Pathol 2004;14:164-174.

19. Takata F, Dohgu S, Matsumoto J, et al. Brain pericytes among cells constituting the blood-brain barrier are highly sensitive to tumor necrosis factor- $\alpha$, releasing matrix metalloproteinase- 9 and migrating in vitro. J Neuroinflammation 2011;8:106. doi: 10.1186/1742-2094-8-106.

20. Sato W, Tomita A, Ichikawa D, et al. CCR2 ${ }^{+} \mathrm{CCR} 5^{+} \mathrm{T}$ cells produce matrix metalloproteinase- 9 and osteopontin in the pathogenesis of multiple sclerosis. J Immunol 2012; 189:5057-5065.

21. Olmos G, Lladó J. Tumor necrosis factor alpha: a link between neuroinflammation and excitotoxicity. Mediators Inflamm 2014;2014:861231. doi: 10.1155/2014/861231.

22. Haacke EM, Makki M, Ge Y, et al. Characterizing iron deposition in multiple sclerosis lesions using susceptibility weighted imaging. J Magn Reson Imaging 2009;29:537544.

23. Langkammer C, Scheurer F, Krebs N, et al. Quantitative susceptibility mapping (QSM) as a means to measure brain iron? A post mortem validation study. Neuroimage 2012; 62:1593-1599.

24. Sbardella E, Tona F, Petsas N, Pantano P. DTI measurements in multiple sclerosis: evaluation of brain damage and clinical implications. Mult Scler Int 2013;2013: 671730. doi: 10.1155/2013/671730.
25. Qian W, Chan Q, Mak H, et al. Quantitative assessment of the cervical spinal cord damage in neuromyelitis optica using diffusion tensor imaging at 3 Tesla. J Magn Reson Imaging 2011;33:1312-1320.

26. Yu CS, Lin FC, Li KC, et al. Diffusion tensor imaging in the assessment of normal-appearing brain tissue damage in relapsing neuromyelitis optica. AJNR Am J Neuroradiol 2006;27:1009-1015.

27. Ciccarelli O, Barkhof F, Bodini B, et al. Pathogenesis of multiple sclerosis: insights from molecular and metabolic imaging. Lancet Neurol 2014;13:807-822.

28. Bertholdo D, Watcharakorn A, Castillo M. Brain proton magnetic resonance spectroscopy: introduction and overview. Neuroimaging Clin N Am 2013;23:359-380.

29. Stromillo ML, Giorgio A, Rossi F, et al. Brain metabolic changes suggestive of axonal damage in radiologically isolated syndrome. Neurology 2013;80:2090-2094.

30. Rovira A, Alonso J. 1H magnetic resonance spectroscopy in multiple sclerosis and related disorders. Neuroimaging Clin N Am 2013;23:459-474.

31. Llufriu S, Kornak J, Ratiney H, et al. Magnetic resonance spectroscopy markers of disease progression in multiple sclerosis. JAMA Neurol 2014;71:840-847. doi: 10.1001/ jamaneuro.2014.895.

32. Azevedo CJ, Kornak J, Chu P, et al. In vivo evidence of glutamate toxicity in multiple sclerosis. Ann Neurol 2014; 76:269-278. doi: 10.1002/ana.24202.

33. Choi IY, Lee SP, Denney DR, Lynch SG. Lower levels of glutathione (GSH) in the brains of secondary progressive multiple sclerosis patients measured by $1 \mathrm{H}$ magnetic resonance chemical shift imaging at 3 T. Mult Scler 2011;17:289-296.

34. Comabella M, Montalban X. Body fluid biomarkers in multiple sclerosis. Lancet Neurol 2014;13:113-126.

35. Londono AC, Castellanos FX, Arbelaez A, et al. An ${ }^{1} \mathrm{H}-\mathrm{MRS}$ framework predicts the onset of Alzheimer's disease symptoms in PSEN1 mutation carriers. Alzheimers Dement 2014;10: 552-561. doi: 10.1016/j.jalz.2013.08.282.29.

36. Daams M, Geurts JJ, Barkhof F. Cortical imaging in multiple sclerosis: recent findings and "grand challenges". Curr Opin Neurol 2013;26:345-352. doi: 10.1097/WCO. 0b013e328362a864. 


\title{
Neurology \\ Neuroimmunology \& Neuroinflammation
}

\author{
Nonconventional MRI biomarkers for in vivo monitoring of pathogenesis in multiple \\ sclerosis \\ Ana C. Londoño and Carlos A. Mora \\ Neurol Neuroimmunol Neuroinflamm 2014;1; \\ DOI 10.1212/NXI.0000000000000045
}

This information is current as of November 20, 2014

$\begin{array}{ll}\begin{array}{l}\text { Updated Information \& } \\ \text { Services }\end{array} & \begin{array}{l}\text { including high resolution figures, can be found at: } \\ \text { http://nn.neurology.org/content/1/4/e45.full.html }\end{array} \\ \text { References } & \begin{array}{l}\text { This article cites } 35 \text { articles, } 3 \text { of which you can access for } \\ \text { http://nn.neurology.org/content/1/4/e45.full.html\#\#ref-lis }-1\end{array} \\ \text { Permissions \& Licensing } & \begin{array}{l}\text { Information about reproducing this article in parts (figures, } \\ \text { its entirety can be found online at: } \\ \text { http://nn.neurology.org/misc/about.xhtml\#permissions } \\ \text { Information about ordering reprints can be found online: } \\ \text { http://nn.neurology.org/misc/addir.xhtml\#reprintsus }\end{array}\end{array}$

Neurol Neuroimmunol Neuroinflamm is an official journal of the American Academy of Neurology.

Published since April 2014, it is an open-access, online-only, continuous publication journal. Copyright $\odot$ 2014 American Academy of Neurology. All rights reserved. Online ISSN: 2332-7812.

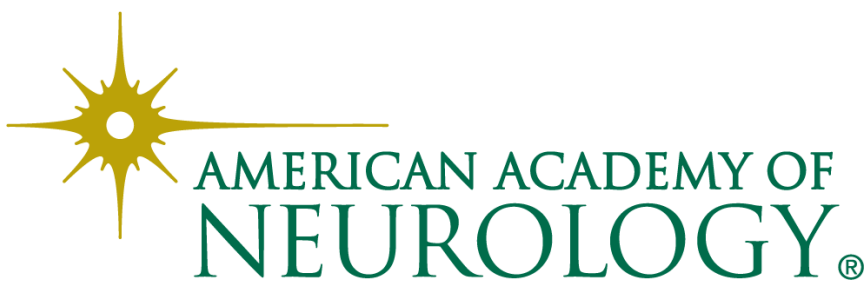

